\title{
GEOMATIC 3D MODELING OF A STATUE (ALSO) FOR STRUCTURAL ANALYSIS AND RISK EVALUATION: THE EXAMPLE OF SAN GIOVANNINO MARTELLI IN FLORENCE
}

\author{
Anna Spangher ${ }^{\mathrm{a}}$, Domenico Visintini ${ }^{\mathrm{a}}$, Grazia Tucci ${ }^{\mathrm{b}}$, Valentina Bonora ${ }^{\mathrm{b}}$ \\ ${ }^{\text {a }}$ University of Udine - DPIA, Polytechnic Department of Engineering and Architecture, via delle Scienze 206, Udine, Italy, \\ anna.spangher@gmail.com, domenico.visintini@uniud.it \\ ${ }^{\mathrm{b}}$ GECO Lab., University of Florence - DICeA, Dept. of Civil and Environmental Engineering, via P.A. Micheli 8, Florence, Italy, \\ grazia.tucci@unifi.it, valentina.bonora@unifi.it
}

KEY WORDS: Laser scanning, 3D Modeling, Cultural Heritage, Structural Analysis, Level of Detail, Finite Element Method

\begin{abstract}
:
This work has been developed among the researches of a $\mathrm{PhD}$ thesis in Civil and Environmental Engineering and Architecture of the University of Udine in cooperation with the GECO Laboratory of the University of Florence. It focuses on the interaction between Geomatics and Structural Analysis, both applied to cultural heritage, and expressly to artefacts and structures in stone materials, like the case study of this paper, the marble statue called "San Giovannino Martelli" (Saint John the Baptist) conserved in Florence.

At the beginning, some interesting examples of surveying and structural analyses on statues are reported, in order to remind the complementary tasks and requirements of geomatics and structural analysis. Current laser scanning systems can accurately survey the geometry of a statue or any cultural heritage artefact, essential to understand their structural behaviour and resilience capability.

Afterwards, following the few Italian regulations in this field, the possible risks of museum goods are described: topics of this part are more familiar for structural engineers as object classification, seismic reactions, damage mechanisms, possible movements (adherent, slipping and oscillation), dynamic domains, anyway necessary steps to evaluate the risk and so to define eventual interventions.

The artistic description of the statue, its debated attribution to Donatello or/and to Desiderio da Settignano and its history is later recalled, remembering that the surveying has been done for the idea to $3 \mathrm{D}$ print a replica and to place it in the original place. Having used a close range laser scanner, the obtained 3D model has an impressive geometrical Level of Detail (LoD), whose geometric features are explained in the paper, underlying that such extremely detailed mesh is directly given as output from the laser scanner software. The model simplifications by four decimation are therefore explained and also changes to geometry, like shifts on centre of the mass or barycentre with respect to the original one, are evaluated: since these are pretty null, all the models can be used for structural analysis. Software Scan-and-Solve, a Rhinoceros plug-in, has been employed for Finite Elements Method (FEM) analysis, considering the sole weight and also a horizontal force, as a seismic event or an accidental push, that can synthesize the possible statue risks. The force intensity and geometry have been computed considering the resistance to the overturning for the adherence among statue and pedestal. The more numerically accurate results has been obtained with the more simplified model, having only $7 \%$ of initial triangles, since this situation better exploits the computational resources for solver precision and for congruent geometrical LoD and FEM resolution.
\end{abstract}

\section{INTRODUCTION}

This work is the result of the collaboration between the University of Udine and the University of Florence, occurred in a research labour carried out within a PhD research in Civil and Environmental Engineering and Architecture. The main topic of this research was properly the investigation on what could be the possible contribute of Geomatics to Structural Engineering. This argument is strictly correlated to the theme of the Conference since it results evident that, in order to correctly evaluate the risks a cultural heritage good is exposed to and to define as appropriately as possible its resilience capability, it is necessary to analyse also its structural behaviour. It is clear that a right approach to structural analysis includes, beside the knowledge of material characteristics, loads and actions applied and existing restraints, also a modelling as realistic as possible of the object geometry. This last aspect has not been deeply developed, especially because structural analysis is mainly implemented on new buildings, generally characterized by simple and symmetrical shapes, easy to reconstruct by geometric primitives. This situation dramatically changes when the objects to analyse are historical buildings or artefacts, where geometry results irregular, more complex, not symmetrical, with many undercuts and differences in thickness, heights and openings. Here comes into play the essential role of the geomatics, very well known in our scientific community; anyway some methodological aspects and examples of surveying of the cultural heritage can be found also in Gonizzi Barsanti, Remondino, Visintini (2013) and in Balletti and Guerra (2015). Possible contributions of the geomatics to specifically manage cultural heritage exposed to risk are instead described in Tucci and Bonora (2015). Building dimensional data contained in 2D technical representation from geomatic surveying are thus the base on which structural engineers perform their Finite Elements Method (FEM) structural analysis.

Much more difficult is the situation when the object to structurally analyse is a statue or a sculpture work: 2D representations are no more sufficient to such aim and a precise 3D model, usually realized for 3D navigations or nowadays of 3D prints, becomes now mandatory.

The most famous and cited example of the geomatics contribution to statues structural analysis is for sure the 3D model of the Michelangelo's David produced by the Stanford University (Levoy et al., 2000). Importing such a model in the software Ansys, a well-known FEM software, a static and a seismic verification were performed (Borri and Grazini, 2006). In particular, several different supposed inclinations were 
considered, in order to define the reason why the back support, called broncone, and the left ankle of the statue resulted cracked, first example of risk on a statue simply due to its own weight. So far, researches on structural analysis performed on 3D models derived from laser scanning surveying have not been so frequent, also because few models have an unrestricted use; therefore, the possibility to use freely the model of Michelangelo's David of the Stanford University, along with its artistic and cultural importance, made it one of the most studied examples. Among all, it deserves to cite the works of Lolli (2010) and of Pascale, Bastianini, Carli (2011). As logical, the largest part of the published work on this argument relates to the most famous arts object, since it is natural that the major effort for saving and preserving is done on these kinds of objects. Therefore, another important study is the one held on the Bronzes of Riace statues by De Canio (2012). In this case, the analysis was dedicated to the possible introduction of seismic base isolations for the statues. A particularly interesting work is that of Riccardelli et al. (2014) held on the Tullio Lombardo's statue of Adam, preserved in the Metropolitan Museum of Art in New York (USA) which crashed because of the collapse of its pedestal in 2002. In order to design the restoration and with the purpose to find a less invasive and more reversible approach of reconstruction, the conservators decided to preliminary study all the aspect laser scanning the fragments of the statue, virtual reconstructing the model of the statue and finally analysing it with FEM. The last example considered was the work of Sorace and Terenzi (2015) which concerns the evaluation of seismic response of statues exhibited in art museums and a strategy of baseisolated floor for their enhanced protection. The analysed statue is a marble copy of a Hellenistic sculpture of the Greek philosopher Socrates: in this case, the structural analysis has been carried out onto a simplified 3D model of the statue.

These examples confirm the essential function of the geomatics to produce accurate and detailed 3D models of statues; in particular, current triangulation laser scanners can excellently detect forms of any level of complexity, as are surfaces of sculptures or of small museum goods. As known, photogrammetry is another geomatic technique producing $3 \mathrm{D}$ points clouds, since nowadays is carried out following Structure from Motion approach but, for the case of statues, the surface generally with little colour variations could make difficult the fundamental steps of image matching.

\section{STRUCTURAL ANALYSIS OF MUSEUMS GOODS}

Differently than the case of buildings and architectural details, which structural aspects have been widely debated all over the world and which standards and regulations have been enacted in almost all countries, less protocols have been produced to define the structural risk of "small size" elements like statues. Referring to Italian rules, few indications on the general procedures to follow are given in "Linee Guida per la valutazione e riduzione del rischio sismico del patrimonio culturale" ( "Guidelines for seismic risk assessment of cultural heritage") (2010), but also this document is mostly oriented to the analysis of existing buildings and does not consider statues, standing alone monuments or isolated artefacts.

The most complete reference on goods of artistic value, even if not implemented in any specific rule or standards, is a document called "Linee Guida per la salvaguardia dei beni culturali dai rischi naturali" ("Guidelines for the Safeguard of Cultural Heritage against Natural Risk") (2005): in this document, the third section is dedicated to museum goods. The standard proposed through this documents considers the risk evaluation and reduction, following this order:
- classification of the objects based on their seismic reaction;

- valuation of the seismic reaction;

- proposal of intervention, according to the previous valuation.

Museum goods are therefore classified into six categories depending on their description: small objects with flat base, small objects with no flat base, statues, sculptures in general and large pots, pictures and paintings in general, chandeliers and hanging objects and other objects. A further classification considers three main categories according to the type of support: objects leaning on a flat surface (on floor, on pedestal, in a showcase, on shelves, on wall, from ceiling), fixed objects (on a flat surface or on a pedestal) and hanging/suspended objects (on a wall or from ceiling).

These two classifications are functional to the definition of:

- the possible mechanisms of dynamic reaction (adherent movement, slipping movement or oscillation movement), and therefore the risks the goods are exposed to;

- the possible damage mechanisms (over stressing, over displacements, repeated collision), which are defined according to the category of support or, otherwise, to the category of the object.

Once defined the kind of reactions and damage mechanisms acting, the guidelines propose different approaches to possible solutions for preserving and safeguarding the objects.

In this research, the focus is given to the determination of which dynamic reactions, and so which damage mechanisms, occur on a statue, varying the forces applied. It is then previously necessary to define when each dynamic reaction will be activated on an object of mass $M$, and specifically:

- adherent "movement": will occur when the relative motion between the object and its support will result null. The force applied to the object of mass $M$ will then result equal to:

$$
F=M a_{a}=M a_{g}
$$

being $a_{a}$ the absolute acceleration of the object and $a_{g}$ the dragging acceleration, applied to the support.

- slipping movement: will start instead when the force of inertia $M a_{g}$, transmitted by the support to the object in conditions of perfect adherence, exceeds (in absolute value) the frictional resistance of the first detachment, i.e. when:

$$
a_{g}>\mu g
$$

being $g$ the gravity acceleration and $\mu$ the friction coefficient between the object and the support/plane surface.

The force transmitted to the object during this kind of motion will be (substituting (2) in (1)) therefore:

$$
F=M a_{g}=M \mu g
$$

- oscillation movement: will occur when the slipping movement is, for some reason, prevented and the rocking motion will be initiated. Following the West's formula the acceleration necessary to initiate a rocking motion will be:

$$
a_{g}=\frac{B}{H} g
$$

being $H$ the height of the centre of mass $G$ on the plane and $B$ the distance of the projection of $G$ from the rotation axis, coincident with the base border $\left(O\right.$ or $\left.O^{\prime}\right)$ (see Figure 1).

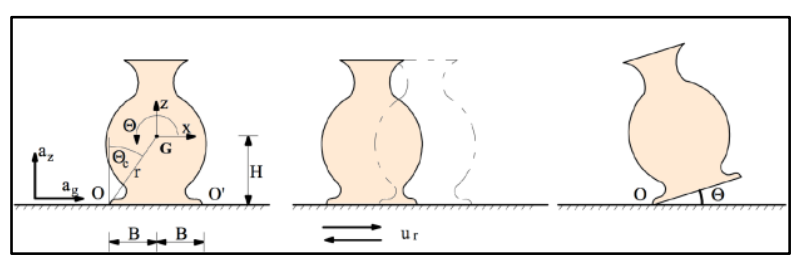

Figure 1. Object and its slipping and oscillation movements. 
It will result therefore that the condition for having an oscillation movement is that the friction coefficient has to be greater that the breadth-height-ratio. While, in order to overturn the object, it must be applied an acceleration of intensity at least equal to $a_{g}$ for a time sufficient to ensure that the speed of the object reaches a critical value.

In order to study the type of movement that could affect the object, it is convenient to assume simplified criteria, like the ones proposed by Ishiyama (1982) considering the overturning of lean rigid bodies through the imposition of conditions in the intensity of acceleration, velocity and displacement. Through the analysis of these impositions it is therefore possible to determinate three main domains (Figure 2):

- Domain A: which corresponds, considering equation (4), to values of ratio $\frac{B}{H}>\frac{a_{g}}{g}$, and therefore with no relative movement because $\mu>\frac{a_{g}}{g}$, considering equation (2).

- Domain B: which corresponds to a condition of oscillatory motion, but at an insufficient speed to cause the overturning.

- Domain C: which corresponds to a condition for which it is likely to occur tipping.

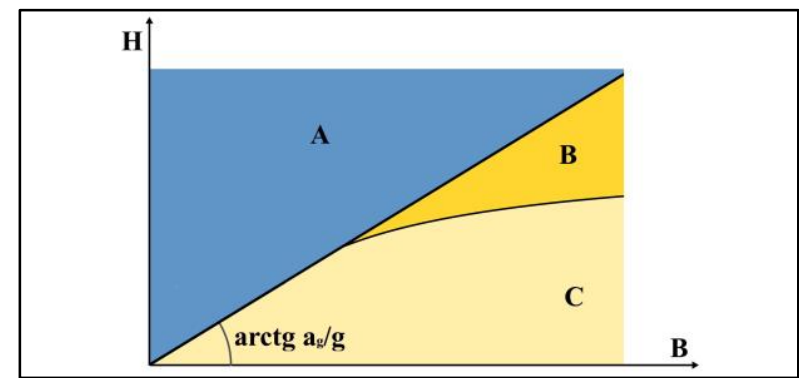

Figure 2. Domains of Ishyiama of possibile movement risks.

In light of the foregoing, it is evident that, to fully analyse all the structural risks of an object, it is necessary to know:

- the position of its centre of gravity,

- the condition relating to its connections with the boundary (leant, fixed, suspended, ...),

- the conditions of the resistance of the object material,

- the damages or cracks suffered,

- the forces that might impact on the object.

Last point is crucial for the risk evaluation of museum goods since, apart the own weight, seismic forces or accidental push could be the more dangerous loads on their structural resistance.

\section{THE TEST CASE OF SAN GIOVANNINO MARTELLI IN FLORENCE}

The statue of "San Giovannino Martelli", also known as Saint John the Baptist, Saint John Martelli, Saint John the Child Martelli, is a full-figure sculpture currently preserved in the Bargello Museum of Florence, represents the case of interest for this research. Its name is due to the Martelli family, which contracted and owned it until 1913, year of its donation to the famous museum institution. The commission, which allegedly occurred between 1455 and 1460, is testified by several historical documents. For sure, one the most important is the mention in "The lives of the most excellent painters, sculptors and architects" by Vasari, where the statue is reported as one of Donatello's works owned by the Martelli family. Actually the attribution to a definite author is still uncertain and debated among art historians, who are partly inclined to consider Donatello as the sole executor, partly instead prefer for the hypothesis of Desiderio da Settignano as the author, as a student of the first, or, at last, it was also supposed for a collaborative work between the two artists, since peculiarities of both of them come to light from the study of the sculpture. The young man represented is no more than ten or twelve years old, but it seems too much and badly developed, with feet and hands so big and rather high, resulting equal to $1,52 \mathrm{~m}$ from the performed surveying. He is set in the act of walking to the left, with the step just opened, with the left leg, on which the body rests, not stuck solidly but a bit tilted in the direction of the path, and his foot sinking the thumb into the ground, while the right leg participates with reluctance to the movement, so that the foot is all still adhering to the ground. The same fatigue is shown in the arms: the right falls down the side, calling support, the hand holding the cross with three fingers and the stone of penance folded with the other two; the left, also close to the body, tightens only with his thumb and index finger, and not even strongly, the folder where the other fingers lie. The mantle of goatskin, which goes down to the knees, fastens with his end on the right side, that is narrow at the waist, with a woven cloth, and the mantle released hanging, as something insignificant, from the left shoulder cannot hide the delicacy body, tapered, with sloping shoulders, with skinny chest on which he plunges his neck (De Nicola, 1913).

The statue, realized in marble, even if in full-figure, was probably set on a pedestal against a wall, as it is possible to see in the right part of a painting representing the Martelli family and dated 1777. Since it was always conserved in closed places, it has a perfect state of preservation and only the gilding decayed because of the time. Currently it is conserved in the "Hall of Donatello and the fifteenth century sculpture" at the Bargello Museum and is located on a pedestal, close to the most famous bronze of Donatello's David.

\section{FROM DATA ACQUISITION TO 3D MODEL}

Referring to the object of interest and having the possibility to test a close range laser scanner, it resulted logical to adopt this particular device, very useful for recording small objects and features requiring highly detailed modeling. The device used was the optical triangulation laser scanner Nikon ModelMaker MMDx100 Handheld (Figure 3), characterized by an accuracy of $10 \mu \mathrm{m}$ and having also a probe contact sensor.

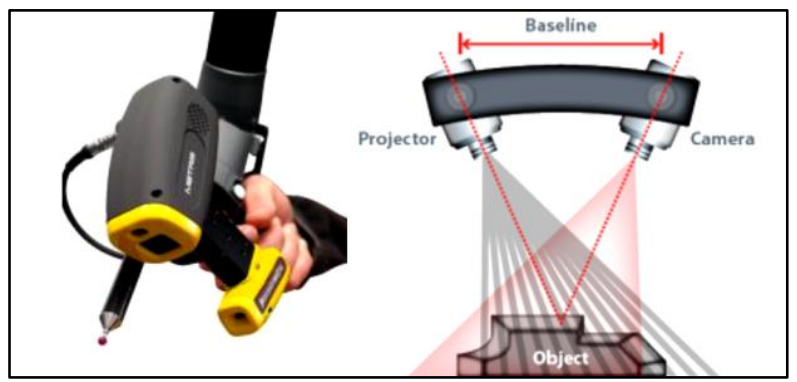

Figure 3. Nikon MMDx100 scanner used for the surveying.

Such laser scanner was integrated into the Nikon MCAx25+ Manual Coordinate Measuring Arm, which is a reliable and easy-to-use portable 7-axis articulated arm, with a point repeatability of $27 \mu \mathrm{m}$. The surveying of the sculpture was executed by two operators of Leonardo 3D Metrology, during the course "Digitalization of archaeological finds and works of plastic art with scanner triangulation" held at the Geomatics Laboratory for Conservation and Communication of Cultural Heritage of the University of Florence.

The family of instruments defined by the term "Articulated Arms of Measurement or Anthropomorphic Measuring Arms" or even by the acronym AACMM, namely "Articulated Arm Coordinate Measuring Machines" allow to fit best the specific 
needs of high flexibility of movement useful to survey complex geometries, mainly where are undercuts characterize the surface. AACMM are manually controlled by operator and work through the collection of 3D points of the surface. They are typically constituted by three rods, connected together at their ends by joints, which allow rotational movements, which give rise to five, six or even seven degrees of freedom. A joint is placed at the lower end of the first segment and connects it to the support base, while the end of the second segment is a third coupling that allows the mounting of a spherical probe. This last allows to measure directly by contact the position of some "control points", which are indispensable to when the instrumentation or the object has to be moved. Each joint has two angular encoders: the set of angular positions of all the encoders, associated to the relative lengths of the segments, allows the calculation of the $\mathrm{X}, \mathrm{Y}, \mathrm{Z}$ coordinates of the tip of the contact element and/or of the centre of the laser scanner.

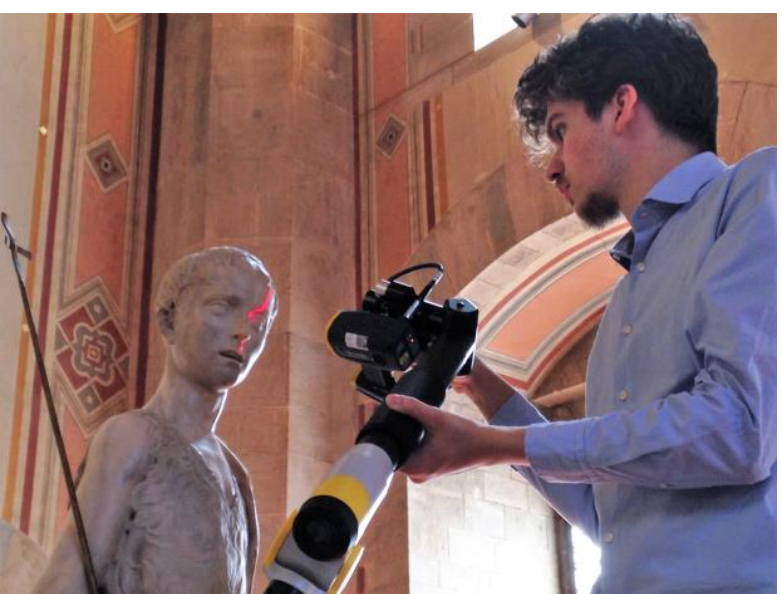

Figure 4. Surveying of the St. John the Baptist statue by

Nikon MMDx100 onto Nikon MCAx25+ measuring arm.

The surveying of the statue of St. John the Baptist arose as a part of the project "Digital technologies for the documentation, management and divulgation of cultural heritage in the Museum of Casa Martelli in Florence", held between the Superintendence for the Historical, Artistic and Ethnoanthropological Heritage of the Museums of City of Florence and the Geomatics Laboratory for Conservation and Communication of Cultural Heritage of the University of Florence, and had as its main goal, the purpose to use the reprinted 3D model, or otherwise the virtual model, as a replica in the Museum of Casa Martelli, its original location. Today, 3D printing (e.g. Tucci and Bonora, 2011) is a very popular topic, whose reproduction likelihood depends either from the surveying accuracy (if done) or from printing technology and material. For this statue, the model had to be extremely precise, namely with the maximum geometrical Level of Detail (LoD). The acquisition operations took about four hours of work, moving the instrumentation around the statue and exploiting the positioning by probe of 18 control points to solve the clouds alignment. All the sculpture surface was scanned with MMDx 100 and the 3D points cloud was directly processed by the software Geomagic Wrap, which automatically allows clouds alignment and transforms the points into a 3D surface. The process is very rapid but is not easily controllable by the user. In truth, the well advantage of this "black box" is the automatic creation of an extremely dense 3D mesh, not so easy process for complex objects and/or from points clouds acquired by classic "topographic" laser scanners. Furthermore, such surface is yet topologically correct, namely without nonmanifolds, double or self-intersecting triangles.
Summarizing, St. John the Baptist statue/model has a bounding box of $0,459 \mathrm{~m}$ ( $\mathrm{X}$ along right viewing the statue), $0,366 \mathrm{~m}$ ( $\mathrm{Y}$ coming out back) and 1,591 $\mathrm{m}$ ( $\mathrm{Z}$ along the vertical), where such $\mathrm{X}$ and $\mathrm{Y}$ values are those of the base. The surface of $2,0656 \mathrm{~m}^{2}$ is composed by a mesh of 5.178 .132 triangles, each one having a mean area of $0,40 \mathrm{~mm}^{2}$ and then a mean edge of only $0,95 \mathrm{~mm}(!)$, i.e. an impressive LoD (Figure 5 , first column). To obtain a closed surface, and therefore a 3D solid model, a simple operation was required, consisting in closing the only hole, given by the base of the statue, exactly its lower part adherent to the pedestal, obviously not scanned.

\section{SIMPLIFICATION OF THE 3D SOLID MODEL}

As just written above, the main purpose of this surveying was a 3D model for reprinting or virtual reality, with maximum geometrical LoD while, as reported in the Introduction, such high resolution is not necessary, on the contrary, it will led to an excessive computation of the FEM analysis software. It is then necessary a simplification of the $3 \mathrm{D}$ mesh, paying attention to not change some important geometrical values, like the coordinates of the centre of mass or the total volume of the object, which have a critical importance, as described in paragraph 2. There are several algorithms that lead to the simplification of a mesh, but all of the techniques proposed in literature are based on some variation or combination of two primitive basic mechanisms: the union of triangles vertices and the decimation of the meshes. The first method relies on the use of clustering algorithms where, by setting up a voxel grid, it is possible to combine the vertices lying in the same voxel. These algorithms provide only limited control over the complexity, topology or the quality of the resulting mesh and, therefore, are not suitable for this purpose case (Bischoff and Kobbelt, 2004). The decimation instead describes that class of algorithms that allows obtaining a mesh, with fewer faces, edges and vertices, by iterative processes which are based on the removal of the "less important" vertices and faces relating to them and on re-triangulation of the resulting hole. Such algorithms allow keeping the main edges of the model and therefore are the most suitable for the purposes that we are prefixing.

In order to find a balanced geometrical LoD of the model exploitable for structural analysis, four models were created with Geomagic Wrap, by decreasing the number of triangles composing their meshes. Figure 5 shows such models, as well as the original one yet described in paragraph 4, with the corresponding main geometric values and differences. As seen in paragraph 2 , centre of mass $G$ is fundamental for possible movements and then its position shifts due to decimations have to be checked. Also the barycentre $\mathrm{C}$ of the mesh, differing from $\mathrm{G}$ since it is considered as an empty surface, has been computed for each decimation.

The XYZ coordinate system has origin at the centre of the base, having a mean width of $7 \mathrm{~cm}$ : for such reason, St. John the Baptist is $1,52 \mathrm{~m}$ tall. Due to its particular posture, the statue centre is just a little to the right $(X=+34,7 \mathrm{~mm}$ for $\mathrm{G}$ and $\mathrm{X}=+31,7 \mathrm{~mm}$ for $\mathrm{C}$ ) and to the back ( $\mathrm{Y}=+21,4 \mathrm{~mm}$ for $\mathrm{G}$ and $\mathrm{Y}=+21,3 \mathrm{~mm}$ for $\mathrm{C})$. Regarding centre elevation $(\mathrm{Z}=$ $+722,9 \mathrm{~mm}$ for $\mathrm{G}$ and $\mathrm{Z}=+660,1 \mathrm{~mm}$ for $\mathrm{C}$ ), coordinate values are less than half of the total height, then the lower part has more volume/mass, as usual in the majority of sculptures.

The first simplified model was obtained by reducing of $50 \%$ the number of the triangles of the mesh: consequently, the medium length of mesh edges grows up to $1,31 \mathrm{~mm}$. The position of the centre of mass remains "exactly" the same $(0,001 \mathrm{~mm}$ shift along vertical $\mathrm{Z}$ !), while barycentre position grows of $4,4 \mathrm{~mm}$ along vertical $\mathrm{Z}$. 
The International Archives of the Photogrammetry, Remote Sensing and Spatial Information Sciences, Volume XLII-5/W1, 2017 GEOMATICS \& RESTORATION - Conservation of Cultural Heritage in the Digital Era, 22-24 May 2017, Florence, Italy

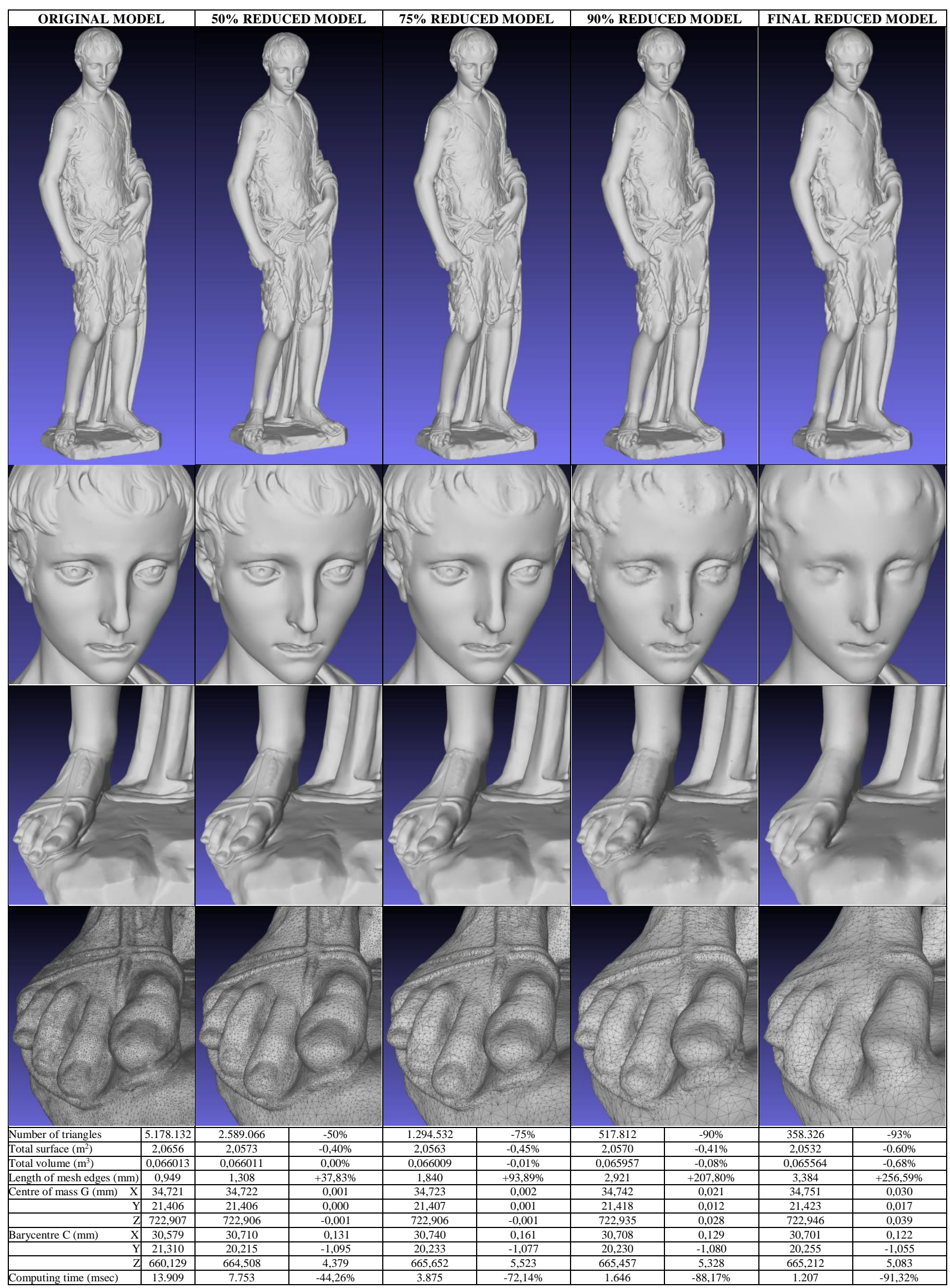

Figure 5. Five models of the statue with different reduction percentage: comparison of geometrical differences and computing time. 
A second decimation was performed reducing the number of the triangles up to the $75 \%$, obtaining edges with a medium length equal to $1,84 \mathrm{~mm}$; considering the original values, also in this case, variations of centre of mass position are null (numerically $0,002 \mathrm{~mm}$ along $\mathrm{X}$ ) while barycentre $\mathrm{Z}$ coordinate changes of $5,5 \mathrm{~mm}$. Another model was produced with a reduction of the $90 \%$ of the triangles, with a consequent medium length of mesh edges equal to $2,92 \mathrm{~mm}$. The fourth and last simplified model has only the $7 \%$ of the original number of triangles of the mesh, with a medium length of mesh edges equal to $3,38 \mathrm{~mm}$. The most interesting aspect is that also for these last two strong decimations, the position of centre of mass is basically the same of the original model. Finally, considering all five mesh models, variations in volume are null (as the mass centre position), a part an increase of $0,68 \%$ for the fifth one that anyway can be neglected.

As last remark of these decimations is the use of the "Mesh doctor" command to ensure topologically corrects triangles.

\section{STRUCTURAL ANALYSIS AND RISK EVALUATION}

Being the object of interest a statue leaning on a flat surface, on a pedestal, the possible mechanisms of dynamic reaction, and correlated damage mechanisms, according to what reported in paragraph 2, could be the following movements:

- adherent, with possible over stressing;

- slipping, with possible over displacement;

- oscillation, with possible repeated collision and overturning.

Starting from the geometry of the 3D model obtained (the original one or those with lower geometrical LoD), the goal is to known which one of the above mechanism will occur on the statue, in order to evaluate the risks it is exposed. In this way, once defined the actions operating and their effects, it will be possible to outline how to plan protection strategies in order to not only preserve the cultural object but to increase its resilience capability. For this aim, a particular software for FEM structural analysis that is Scan-and-Solve ${ }^{\mathrm{TM}}$ ver.1.6 (Intact Solutions, 2014) has been used. Although its particular name recalls scan activities, its main peculiarity is to work directly within a 3D modeling environment software since it is no other than a plug-in on the well-known program Rhinoceros. This makes possible to straightaway exploit the 3D model outputted from the surveying (but also from other data) and so to-solve the structural analysis, obviously with some simplification. The only required condition is that the surface model has to be closed and topologically correct, thus constituting a solid model. In fact, Scan-and-Solve adopts a completely different principle, automatically computing the structural geometry by suitably subdividing the solid model in a finite number of small ashlars. This approach is completely different from those of the classical FEM software, where the structure geometry has to be strongly simplified and/or rearranged, namely there is a structural $3 D$ modeling before to perform any computation. For such aim, an interesting semiautomatic procedure has been recently proposed by Castellazzi et al. (2015). Scan-and-Solve allows instead to use the object geometry without any re-modeling phase, with a great saving of time and also with the advantage of preserving from errors due to interpretation of the geometry (Freytag, Shapiro, Tsukanov, 2011). To proceed with the structural analysis, it is necessary to previously define some aspects:

- mechanical characteristic,

- restraints,

- forces acting,

- parameters of FEM analysis.
In Visintini and Spangher (2014) the choices and some details of the previous aspects are explained for the similar case of the marble statue of Emperor Claudio preserved in Aquileia.

For the St. John the Baptist example, concerning its material, taking into consideration that no characterization tests were carried out, mechanical properties were established by referring to typical values suggested in literature, particularly adopting coefficients of a similar case treated by Sorace and Terenzi (2015) and reported in the following Table 1.

\begin{tabular}{|ccccccc|}
\hline Description & $\begin{array}{c}\text { Density } \\
\left(\mathrm{kg} / \mathrm{m}^{3}\right)\end{array}$ & $\begin{array}{c}\text { Elastic } \\
\text { modulus } \\
(\mathrm{MPa})\end{array}$ & $\begin{array}{c}\text { Poisson } \\
\text { Ratio }\end{array}$ & $\begin{array}{c}\text { Default } \\
\text { failure } \\
\text { criterion }\end{array}$ & $\begin{array}{c}\text { Ultimate } \\
\text { tensile } \\
\text { strength } \\
(\mathrm{MPa})\end{array}$ & $\begin{array}{c}\text { Ultimate } \\
\text { compressive } \\
\text { strength } \\
(\mathrm{MPa})\end{array}$ \\
\hline $\begin{array}{c}\text { Marble } \\
\text { Low density }\end{array}$ & 2.650 & $6,00 \times 10^{4}$ & 0,25 & $\begin{array}{c}\text { Mohr } \\
\text { Coulomb }\end{array}$ & 7,00 & 68,9 \\
\hline
\end{tabular}

Table 1. Material characteristcs adopted for the statue.

Regarding the restraints, since it was supposed that no movement on the base of the statue is possible, a fixed end was there adopted. No other restrains were considered.

As it concerns the forces acting, models were analysed with:

- only gravity load,

- gravity load and a horizontal force simulating an accidental push or a seismic action, even if simplified.

The horizontal force applied was assumed as the minimum force necessary to activate an oscillation movement, as described in paragraph 2 . All the five models reported in paragraph 5 have the same volume and position of centre of mass. It is then possible to calculate the horizontal distances between the projections of centre of mass $\mathrm{G}$ and the axis of rotation, according Figure 1, the same for all five models:

$$
\begin{aligned}
& \mathrm{B}_{\mathrm{x}}^{1}=24,18-3,47=20,71 \mathrm{~cm} \\
& \mathrm{~B}_{\mathrm{x}}^{2}=21,69+3,47=25,16 \mathrm{~cm} \\
& \mathrm{~B}_{\mathrm{y}}^{1}=18,63-2,14=16,49 \mathrm{~cm} \\
& \mathrm{~B}_{\mathrm{y}}^{2}=17,97+2,14=20,11 \mathrm{~cm}
\end{aligned}
$$

Therefore, in order to verify which force has to be applied for activating an oscillation movement, the minimum distance $\mathrm{B}$ between the projection of $\mathrm{G}$ and its axes of rotation has to be considered, related to the height $\mathrm{H}$ of $\mathrm{G}$ :

$$
\frac{B}{H}=\frac{B_{y}^{1}}{H}=\frac{16,49 \mathrm{~cm}}{72,29 \mathrm{~cm}}=0,228
$$

whence derives that the minimum acceleration to apply for having an oscillation of the object results:

$$
\mathrm{a}_{\mathrm{g}}=\frac{\mathrm{B}_{\mathrm{y}}^{1}}{\mathrm{H}} \mathrm{g}=0,228 \cdot 9,81=2,24 \frac{\mathrm{m}}{\mathrm{s}^{2}}
$$

Considering that the statue volume is equal to $0,066 \mathrm{~m}^{3}$ and the specific weight $\gamma$ of marble is assumed equal to 2.650 $\mathrm{kg} / \mathrm{m}^{3}$, it results that the mass $\mathrm{M}$ of the statue will be $175 \mathrm{~kg}$. Hence, the force necessary to start an oscillation will be:

$$
\mathrm{F}=\mathrm{Ma}_{\mathrm{g}}=\frac{\mathrm{B}_{\mathrm{y}}^{1}}{\mathrm{H}} \mathrm{g}=175 \mathrm{~kg} \cdot 2,24 \frac{\mathrm{m}}{\mathrm{s}^{2}}=392 \mathrm{~N}
$$

Lastly, as concern the FEM analysis parameters, one of most important can be defined as "structural LoD" and is the dimension of the elementary cubic ashlars reconstructing, as a voxel composition, the whole object. The ideal resolution to adopt is that one for which these ashlars have a size as much as possible similar to the minimum dimension of the object to be structurally analysed. In our case, smaller objects are represented by the elements of the pelt, which, nevertheless, do not assume any structural relevance. We can so define that the size of a finger could fit as minimum structural ashlar, hence the optimal is given by $1 \mathrm{~cm}$ ashlar. Scan-and-Solve allows to fix this size (structural LoD) or alternatively the number of ashlars but, from the 
computational point of view, these opposite values depend to the geometrical LoD of the model. If the mesh is extremely detailed (small triangles), the computation of elementary ashlar become quite impossible if also these are very little. Only the fifth more simplified model (358.327 triangles with mean edge of $3,38 \mathrm{~mm}$ ) has been analysed by means of 83.512 ashlars of $10 \mathrm{~mm}$ size. For the other four models, it was possible to use "only" 10.000 ashlars of $22,3 \mathrm{~mm}$ size. Analysis for gravity load condition do not evidence any particular risk, while more interesting are the results when also the horizontal force of $392 \mathrm{~N}$ is applied from right as defined before. Obtained values are shown in Figure 6, where first row pictures represent the total displacement and the second the Mohr-Coulomb danger level. The range colours of total displacement is from blue $(0 \mathrm{~mm}$ at the base) to red $(0,254 \mathrm{~mm}$ at the top). Mohr-Coulomb values are instead coloured only where exceed the criterion (> 1) and maximum and minimum principal stresses have to be checked. Figure 3 also report numerical values of the five analysis carried out, allowing to understand the obtained results reliability.



Figure 6. Analyses on the five models of the statue considering a horizontal force: comparison of results and FEM parameters. 
For the original 5,2 M triangle model it was necessary to adopt a less precise processing system, swapping from the default iterative $\mathrm{SnS}$ solver to the direct sparse solver (DSS), very fast but less accurate and using a large amount of memory. It is clear that as less accurate is the structural analysis as less reliable will be the obtained results and the possible remedies. As can be seen, reported computing times dramatically change with the number of triangles (geometric LoD), the number of ashlars (structural LoD), the processing system chosen $(\mathrm{SnS}$ or DSS), and the numerical precision adopted.

If we degrade the geometry of the object in order to not distinguish important structural parts anymore or to misplace the centre of the mass or even to modify the geometry of the object, even if we use the most performing structural analysis we will obtain completely wrong data. The solution is therefore given by a correct evaluation of both the geometrical and structural LoD: the geometrical definition has to be maintained, except for those peculiar parts that do not have any influence on structural analysis and, at the same time, the structural definition has to be sufficient to solve in a numerically correct way the analysis.

Coming to the fundamental question of the risk to which the statue is exposed, we can answer that parts where MohrCoulomb criteria value is more than one could collapse, due to the adherence with the pedestal, if there the minimum principal stress exceeds the ultimate tensile strength (7 Mpa) of Table 1. The right leg of St. John is the zone where such values are over such limit: meanly 11,4 MPa for first four models and 7,3 $\mathrm{MPa}$ for the last more simplified, barely exceeding the admissible value. It is very interesting to observe that such strong difference is essentially due to structural LoD obliged to adopted for computational reasons: $22,3 \mathrm{~mm}$ for the firsts and $10 \mathrm{~mm}$ for the last, namely 10.000 vs 83.512 ashlars. Stress tensile values obtained by means of better FEM computations parameters evidence a "little" risk, while by considering numerically simplified FEM solutions, an "overestimated" risk comes out, with strength 56\% higher. Nevertheless and fortunately, the application of a $392 \mathrm{~N}$ force (around $40 \mathrm{~kg}$-force) at $72 \mathrm{~cm}$ from the base cannot be the situation of an accidental push, e.g. due to visitors overcrowding, at least since the pedestal is $80 \mathrm{~cm}$ height.

\section{CONCLUSIONS}

This paper presented how a 3D model of a statue coming out from laser scanning surveying can be exploited to evaluate the risk on which is exposed, developing as case study the marble statue of San Giovannino Martelli sculptured by Donatello or Desiderio da Settignano and preserved at the Bargello Museum in Florence. The extremely high LoD of the 3D model (5,2 M triangles) gained by a triangulation scanner onto a measuring arm (to print a replica) is surely exceeding for structural purposes. Since its decimation is an automatic process and the used FEM analysis software is a Rhino plug-in, we can however state that structural analysis becomes fairly simple. FEM results with high LoD models are even worse, since computational resources are spent for the structural discretization and not for the analysis. Starting from this "exceptional" model, decimation percentage can reach 93\%; this does not mean that geomatics provides useless data since, most of times, model LoD is that typical of geomatics, indeed it is what only geomatics can guarantee. Concluding, all the 3D models of the cultural heritage could be used also for structural purposes and risk evaluation, without throwing away precious data: maybe it is only necessary for Geomatics to promote the obtainable output and make it suitable for Structural Engineering inputs.

\section{REFERENCES}

Balletti, C., Guerra, F., 2015. The survey of cultural heritage: a long story. Rendiconti Lincei, 26 (Suppl. 1), pp. 115-125.

Bischoff, S., Kobbelt, L., 2004. Teaching meshes, subdivision and multiresolution techniques. Computer-Aided Design, 36 (14), pp. 1483-1500.

Borri A., Grazini A., 2006. Diagnostic analysis of the lesions and stability of Michelangelo's David. Journal of Cultural Heritage, 7, pp. 273-285.

Castellazzi, G., D’Altri, A. M., Bitelli, G., Selvaggi, I., Lambertini, A., 2015. From laser scanning to finite element analysis of complex buildings by using a semi-automatic procedure. Sensors, 20 (2), pp. 2-20.

De Canio, G., 2012. Marble devices for the base isolation of the two Bronzes of Riace: a proposal for the David of Michelangelo. The 15th World Conference on Earthquake Engineering. Lisboa, Portugal.

De Nicola, G., 1973. Donatello "Il San Giovannino Martelli". Bolletino d'Arte, 7, Roma, Italy, pp. 275-290.

Freytag, M., Shapiro, V., Tsukanov, I., 2011. Finite element analysis in situ. Finite element in Analysis and Design, 47 (9), pp. 957-969.

Gonizzi Barsanti, S., Remondino, F., Visintini, D., 2013. 3D surveying and modeling of archaeological sites - some critical issues. ISPRS Annals of the Photogrammetry, Remote Sensing and Spatial Information Sciences, 2 (5/W1), pp. 145-150.

Intact Solutions, Inc., 2014. Scan-and-Solve for Rhino. http://www.scan-and-solve.com/

Ishiyama, Y., 1982. Motions of rigid bodies and criteria for overturning by earthquake excitations. Earthquake Engineering and Structural Dynamics, 10, pp. 635-650.

Levoy et al., 2000. The digital Michelangelo project: 3D scanning of large statues. SIGGRAPH 'OO Proc. of the 27th Conf. on Computer graphics and interactive techniques, pp. 131-144.

Linee Guida per la valutazione e riduzione del rischio sismico del patrimonio culturale, Consiglio Superiore Lavori Pubblici, 2010.

Linee guida per la salvaguardia dei beni culturali dai rischi naturali. ENEA-MIUR, 2005.

Lolli, A., 2010. Monitoraggio per la diagnostica strutturale del David di Michelangelo. Università di Bologna, Italy.

Pascale, G., Bastianini, F., Carli, R., 2011. Monitoring marble cracking in the David by Michelangelo. Art'11 10th International Conference. Florence, Italy, pp. 21-24.

Riccardelli, C., Soultanian, J., Morris, M., Becker, L., Wheeler, G., Street, R., 2014. The treatment of Tullio Lombardo's Adam: A new approach to the conservation of monumental marble sculpture. Metropolitan Museum Journal, 49.

Sorace, S., Terenzi, G., 2015. Seismic performance assessment and base-isolated floor protection of statues exhibited in museum halls. Bulletin of Earthquake Engineering, 13, pp. 1873-1892.

Tucci, G., Bonora, V., 2011. From real to *. "real". A review of geomatic and rapid prototyping techniques for solid modelling in Cultural Heritage field. The International Archives of the Photogrammetry, Remote Sensing and Spatial Information Sciences, 38 (5/W16), pp. 1682-1777.

Tucci G., Bonora V., 2015. Geomatics and management of atrisk cultural heritage, Rendiconti Lincei, 26 (S. 1), pp. 105-114.

Visintini, D., Spangher, A., 2014. Il contributo della Geomatica per l'analisi strutturale dei beni culturali: l'esempio di una statua romana lesionata. Atti della 18 a Conferenza Nazionale ASITA, Firenze, Italy, pp. 1229-1236. 